\title{
OPTIMAL PERIODIC CONTROL OF \\ RETARDED LIENARD EQUATIONS *)
}

\author{
F. Colonius \\ Division of Applied Mathematics \\ Brown University \\ Box F \\ Providence, R.I. 02912, USA
}

This paper is concerned with optimal periodic control problems for retarded Lienard equations of the form

$$
\ddot{x}(t)+f(x(t)) \dot{x}(t)+g(x(t-r))=u(t),
$$

where $r>0$ denotes the length of the retardation. We are looking for periodic controls $u$ and corresponding trajectories $x$ of the same period length minimizing a certain (average) performance criterion. The aim of this paper is (i) to show and illustrate the applicability of the general methods developed in [2], and (ii) this case study uncovers a severe problem in optimal periodic control of retarded equations concerning the determination of the optimal period length. For large retardations, the problem apparently becomes very sensitive with respect to the optimal period length $\tau$ and no useful information about $\tau$ can be drawn out of the Il-Criterion

More specifically, we consider the following problem:

$$
\text { Minimize }-1 / \tau \int_{0}^{\tau} x(s) d s+\alpha /(2 \tau) \int_{0}^{\tau} u(s)^{2} d s
$$

subject to $(1.1)$ and

$$
\begin{aligned}
& x(t)=x(\tau+t), \quad \dot{x}(t)=\dot{x}(\tau+t), \quad t \in[-r, 0] \\
& \int_{0}^{\tau} u(s) d s=0 .
\end{aligned}
$$

Here $\alpha \in \mathbb{R}$ will be chosen later.

*) This research was performed during a visit to the Institut fur Mathematik der Universität Graz supported by a grant from Deutsche Forschungsgemeinschaft. 
The periodicity condition for $x$ is incorporated into (1.3): This condition guarantees that the periodic extensions to the positive reals of $\mathrm{x}$ and $\mathrm{u}$ satisfy the system equation (1.1).

Though we do not have any specific application in mind, the first term in the performance criteria (1.2) is typical for chemical engineering applications where the average output has to be maximized. The second term relates to the "control energy".

A discussion of previous work on optimal periodic control theory and applications can be found in [2]. Work on time optimal control of the (unretarded) Liénard equation to equilibrium is summarized in [1].

Section 2, below, gives a short account of optimality conditions for periodic control of general functional differential systems. Along with the optimal periodic control problem an optimal steady state problem is considered where constant controls $u$ are sought yielding steady states $x$ such that the performance criterion is minimized. Then the so-called n-Criterion allows to discern optimal steady states around which performance can be improved by allowing proper periodic solutions. In this case the steady state is called locally proper.

In section 3, this result is applied to optimal periodic control of retarded Liénard equations while section 4 discusses numerical findings.

\section{The n-Criterion for Functional Differential systems.}

In this section, a general optimal periodic control problem under constraints is formulated and, based on [2], a I-Criterion for properness is given.

$$
\text { Minimize } 1 / \tau \int_{0}^{T} f_{0}(x(t), u(t)) d t
$$

such that

$$
\begin{aligned}
& \dot{x}(t)=f_{1}\left(x_{t}, u(t)\right) \text { a.a. } t \in[0, \tau] \\
& x_{0}=x_{1} \\
& 0=\int_{0}^{\tau} f_{2}(x(t), u(t)) d t ;
\end{aligned}
$$

here $\tau>0$ is fixed, $x_{t}(s):=x(t+s), s \in[-r, 0], u(t) \in \mathbb{R}^{m}$, and $r>0$ is the length of the delay. The maps $f_{0}: \mathbb{R}^{n} \times \mathbb{R}^{m} \rightarrow \mathbb{R}$, $f_{1}: C\left(-r, 0 ; \mathbb{R}^{n}\right) \times \mathbb{R}^{m} \rightarrow \mathbb{R}^{n}, \quad f_{2}: \mathbb{R}^{n} \times \mathbb{R}^{m} \rightarrow \mathbb{R}^{k^{0}}$ are assumed to be $t$ wice 
continuously Fréchet-differentiable, and the controls $u$ are taken in $L_{\infty}\left(0, \tau ; \mathbb{R}^{m}\right)$. Furthermore, we assume that for every initial function $x_{0}=\psi \in C\left(-r, 0 ; \mathbb{R}^{n}\right)$ and every control $u \in L_{\infty}\left(0, \tau ; \mathbb{R}^{m}\right)$, equation $(2.2)$ has a unique absolutely continuous solution $x$.

The corresponding optimal steady state problem has the following form:

$$
\operatorname{Minimize}_{x \in \mathbb{R}^{n}, u \in \mathbb{R}^{m}} f_{0}(x, u)
$$

such that

$$
\begin{aligned}
& 0=f_{1}(\bar{x}, u) \\
& 0=f_{2}(x, u)
\end{aligned}
$$

where $\bar{x} \in \mathrm{C}\left(-\mathrm{r}, 0 ; \mathbb{R}^{\mathrm{n}}\right)$ is the constant function $\overline{\mathrm{x}}(\mathrm{s}):=\mathrm{x}$. Let $\left(x^{0}, u^{0}\right) \in \mathbb{R}^{n} \times \mathbb{R}^{m}$ satisfy (2.6) and (2.7). Linearization of (2.2) and (2.4) gives

$$
\begin{aligned}
& \dot{x}(t)=D_{1} f_{1}\left(\bar{x}^{0}, u^{0}\right) x_{t}+D_{2} f_{1}\left(\bar{x}^{0}, u^{0}\right) u(t), \quad t \in[0, \tau] \\
& 0=\int_{0}^{\tau}\left[D_{1} f_{2}\left(x^{0}, u^{0}\right) x(t)+D_{2} f_{2}\left(x^{0}, u^{0}\right) u(t)\right] d t .
\end{aligned}
$$

The characteristic function of (2.8) is

$$
\Delta(z)=z I-D_{1} f_{1}\left(\bar{x}^{0}, u^{0}\right) e^{z}
$$

where $e^{z}$. denotes the function $\exp (z \theta), \theta \in[-r, 0]$, and $I$ is the $n \times n$ identity matrix

Introduce the function $\mathrm{H}: C\left(-\mathrm{r}, 0 ; \mathbb{R}^{\mathrm{n}}\right) \times \mathbb{R}^{\mathrm{m}} \times \mathbb{R}^{\mathrm{n}} \times \mathbb{R}^{k} \rightarrow \mathbb{R}$ by

$$
H\left(\psi, u, \lambda_{1}, \lambda_{2}\right):=f_{0}(\psi(0), u)+\lambda_{1}^{T} f_{1}(\psi, u)+\lambda_{2}^{T} f_{2}(\psi(0), u) .
$$

Then consider for $\omega \in \mathbb{R}$ the following expressions (here $j:=\sqrt{-1}$ ):

$$
\begin{aligned}
P(\omega) & :=D_{1} D_{1} H\left(\bar{x}^{0}, u^{0}, \lambda_{1}, \lambda_{2}\right)\left(e^{j \omega \cdot}, e^{-j \omega \cdot}\right) \\
Q(\omega) & :=D_{2} D_{1} H\left(\bar{x}^{0}, u^{0}, \lambda_{1}, \lambda_{2}\right)\left(e^{j \omega \cdot}\right) \\
R & :=D_{2} D_{2} H\left(\bar{x}^{0}, u^{0}, \lambda_{1}, \lambda_{2}\right) .
\end{aligned}
$$

We identify $P(\omega), Q(\omega)$, and $R$ with elements in $\mathbb{d}^{n \times n}, \mathbb{C}^{n \times m}$ and $\mathbb{R}^{m \times m}$, 


\section{0}

respectively. Observe that $R$ is symmetric, $P(\omega)$ is Hermitian and $Q(\omega)^{T}=D_{1} D_{2} H\left(\bar{x}^{0}, u^{0}, \lambda_{1}, \lambda_{2}\right)\left(e^{j \omega} \cdot\right)$.

Define the complex mxm matrix $\Pi(\omega)$ by

$$
\begin{aligned}
\Pi(\omega):= & B^{\mathrm{T}} \Delta^{-1}(-j \omega)^{\mathrm{T}} P(\omega) \Delta^{-1}(j \omega) B \\
& +Q(-\omega)^{\mathrm{T}} \Delta^{-1}(j \omega) B+B^{\mathrm{T}} \Delta^{-1}(-j \omega)^{\mathrm{T}} Q(\omega)+R .
\end{aligned}
$$

The matrix $\Pi(\omega)$ is Hermitian. We get the following result.

Theorem 1. Let $\left(x^{0}, u^{0}\right)$ be an optimal solution of the steady state problem $(2.5)-(2.7)$ and assume

(a) with $\omega:=2 \pi / \tau$, the matrix $\Delta(j p \omega)$ is invertible for all $p \in \mathbb{Z}$;

(b) $\mathbb{R}^{n} \times \mathbb{R}^{k}=\left\{\left(D f_{1}\left(\bar{x}^{0}, u^{0}\right)(\bar{x}, u), D f_{2}\left(x^{0}, u^{0}\right)(x, u)\right): x \in \mathbb{R}^{n}, u \in \mathbb{R}^{m}\right\}$;

(c) $C\left(-r, 0 ; \mathbb{R}^{n}\right) \times \mathbb{R}^{k}=\left\{\left(x_{\tau}-\phi, z\right)\right.$ : there exist $u \in L_{\infty}\left(0, \tau ; \mathbb{R}^{m}\right), \phi \epsilon$ $\in C\left(-r, 0 ; \mathbb{R}^{n}\right)$ with $(2.8), x_{0}=\phi$, and $\left.z=\int_{0}^{\tau} D f_{2}\left(x^{0}, u^{0}\right)(x(t), u(t)) d t\right\}$.

Then the following assertions hold:

(i) There exist Lagrange multipliers $\lambda_{1} \in \mathbb{R}^{\mathrm{n}}, \lambda_{2} \in \mathbb{R}^{\mathrm{k}}$ satisfying

$$
\begin{aligned}
& 0=o_{1} H\left(\bar{x}^{0}, u^{0}, \lambda_{1} \tau, \lambda_{2}\right) \\
& 0=o_{2} H\left(\bar{x}^{0}, u^{0}, \lambda_{1} \tau, \lambda_{2}\right) .
\end{aligned}
$$

(ii) Let $\lambda_{1}, \lambda_{2}$ satisfy (i) and suppose that there is $n \in \mathbb{R}^{\mathrm{m}}$ with $n^{t} \pi(\omega) \pi<0$.

Then $\left(x^{0}, u^{0}\right)$ is locally proper, i.e., for every $\varepsilon>0$ there exist $x$ and $u$ satisfying $(2.2)-(2.4)$ with $\left\|x-\vec{x}^{0}\right\|_{\infty}<\varepsilon,\left\|u-\vec{u}^{0}\right\|_{\infty}<\varepsilon$, and

$$
1 / \tau \int_{0}^{T} f_{0}(x(t), u(t)) d t<f_{0}\left(x^{0}, u^{0}\right)
$$

The proof of this theorem follows the same lines as that of 12 , Theorem 6.1]. The additional isoperimetric contraint (2.4) can be treated in a standard manner.

\section{Periodic Control of Retarded Liénard Equations.}

First we want to assure ourselves that indeed for every $\tau$-periodic forcing function $u$ satisfying (1.4) there exists a corresponding 
$\tau$-periodic solution $x$ of (1.1). Formally, this is not necessary for an application of the results in the previous section, in particular, for an analysis of local properness. It suffices that in every neighborhood of an optimal steady state solution $\bar{x}^{0}, \bar{u}^{0}$ there exist periodic solutions $x$, $u$ (yielding better performance).

Nevertheless the following result can be found in the literature 14 , Theorem 4].

Theorem 2. Let $f$ and $g: \mathbb{R} \rightarrow \mathbb{R}$ be continuous and assume that one of the following conditions is satisfied

(i) there exists $m>0$ such that for $|y| \geq m$ one has $y g(y) \leq 0$ and $\lim \inf g(y) / y>-1$;

(ii) there exists $m>0$ such that for $|y| \geq m$ one has $y g(y) \geq 0$ and $\lim _{|y| \rightarrow \infty} \inf g(y) / y<1$.

Then for every $r \in[0,2 \pi l$, the retarded Lienard equation (1.1) has at least one $2 \pi$-periodic solution for $u \in L^{1}(0,2 \pi, \mathbb{R})$ with $\int_{0}^{2 \pi} u(t) d t=0$.

Remark. I do not know if this result remains valid if the length $r$ of the delay exceeds the length of the period.

The control problem (1.1) - (1.4) formalized in section 1 fits into the general framework of section 2 if we define

$$
x_{1}(t):=x(t) \quad x_{2}(t):=\dot{x}(t)
$$

Then equation ( 1.1 ) becomes

$$
\mid \begin{aligned}
& \dot{x}_{1}(t)=x_{2}(t) \\
& \dot{x}_{2}(t)=-f\left(x_{1}(t)\right) x_{2}(t)-g\left(x_{1}(t-r)\right)+u(t)
\end{aligned}
$$

and the boundary conditions are

$$
\left(x_{1}\right)_{0}=\left(x_{1}\right)_{\tau} \quad\left(x_{2}\right)_{0}=\left(x_{2}\right)_{\tau}
$$

while we have to minimize

$$
-1 / \tau \int_{0}^{T} x_{1}(s) d s+\alpha /(2 \tau) \int_{0}^{T} u(s)^{2} d s .
$$


The corresponding steady state problem is

$$
\begin{aligned}
& \text { Minimize }-x_{1}+a / 2 u^{2} \\
& \text { s.t. } \mid \begin{array}{l}
0=x_{2} \\
0=-f\left(x_{1}\right) x_{2}-g\left(x_{1}\right)+u \\
0=u .
\end{array}
\end{aligned}
$$

Clearly, the optimal steady state satisfies

$$
u^{0}=0, x_{2}^{0}=0
$$

and $x_{1}^{0}$ is the solution of

$$
\min -x_{1} \text { s.t. } g\left(x_{1}\right)=0 \text {. }
$$

Under the assumption

$$
g(x) \neq 0 \text { for } x>0 \text { and } g(0)=0 \text {, }
$$

it follows that $x_{1}^{0}=0$.

Linearization of the constraints (3.3) yields

$$
\left(\begin{array}{ccc}
0 & 1 & 0 \\
-f_{x}\left(x_{1}\right) x_{2}-g_{y}\left(x_{1}\right) & -f\left(x_{1}\right) & 1 \\
0 & 0 & 1
\end{array}\right)
$$

and, evaluated at $\left(x_{1}^{0}, x_{2}^{0}, u^{0}\right)=(0,0,0)$

$$
\left(\begin{array}{ccc}
0 & 1 & 0 \\
-\varepsilon_{y}(0) & -f(0) & 1 \\
0 & 0 & 1
\end{array}\right) .
$$

We assume

$$
\mathrm{g}_{\mathrm{y}}(0) \neq 0
$$

The function $H: C\left(-r, 0 ; \mathbb{R}^{2}\right) \times \mathbb{R} \times \mathbb{R}^{3} \rightarrow \mathbb{R}$ is given by 


$$
H(\psi, u, \lambda)=-\psi_{1}(0)+a / 2 u^{2}+\left(\lambda_{1} \lambda_{2} \lambda_{3}\right)\left\{\begin{array}{l}
\psi_{2}(0) \\
-f\left(\psi_{1}(0)\right) \psi_{2}(0)-g\left(\psi_{1}(-r)\right)+u \\
u
\end{array}\right\} .
$$

For notational convenience, we prefer to write $H$ in the form

$$
\begin{aligned}
& H: \mathbb{R}^{2} \times \mathbb{R}^{2} \times \mathbb{R} \times \mathbb{R}^{3} \rightarrow \mathbb{R} \\
& H(x, y, u, \lambda)=-x_{1}+\alpha / 2 u^{2}+\left(\lambda_{1} \lambda_{2} \lambda_{3}\right)\left(\begin{array}{l}
x_{2} \\
-f^{\prime}\left(x_{1}\right) x_{2}-g\left(y_{1}\right)+u \\
u
\end{array}\right)
\end{aligned}
$$

(here $y=\left(y_{1}, y_{2}\right)^{T}$ corresponds to the delayed term).

Then there are Lagrange multipliers $\left(\lambda_{1}, \lambda_{2}, \lambda_{3}\right)^{\mathrm{T}} \in \mathbb{R}^{3}$ satisfying

$$
\left(\begin{array}{lll}
-1 & 0 & 0
\end{array}\right)+\left(\begin{array}{lll}
\lambda_{1} & \lambda_{2} & \lambda_{3}
\end{array}\right)\left(\begin{array}{lcl}
0 & 1 & 0 \\
-g_{\mathrm{y}}(0) & -\mathrm{f}^{\prime}(0) & 1 \\
0 & 0 & 1
\end{array}\right)=0
$$

i.e.,

$$
\begin{aligned}
& -1-\lambda_{2} g_{y}(0)=0 \\
& \lambda_{1}-\lambda_{2} f(0)=0 \\
& \lambda_{2}+\lambda_{3}=0 .
\end{aligned}
$$

Thus

$$
\mid \begin{aligned}
& \lambda_{1}=-g_{y}(0)^{-1} f(0) \\
& \lambda_{2}=-g_{y}(0)^{-1} \\
& \lambda_{3}=g_{y}(0)^{-1}
\end{aligned}
$$

Linearizing the system equation (3.1) around $\left(x_{1}^{0}, x_{2}^{0}, u^{0}\right)=(0,0,0)$ we find

$$
\dot{x}(t)=A_{0} x(t)+A_{1} x(t-r)+B u(t)
$$

with 


$$
A_{0}:=\left(\begin{array}{cc}
0 & 1 \\
0 & -f(0)
\end{array}\right), \quad A_{1}:=\left(\begin{array}{cc}
0 & 0 \\
-g_{y}(0) & 0
\end{array}\right), \quad B:=\left(\begin{array}{l}
0 \\
1
\end{array}\right) .
$$

Next we analyse the characteristic equation for the homogeneous equation (3.8):

$$
\begin{aligned}
& d(\lambda) \\
& :=\operatorname{det} \Delta(\lambda) \\
& =\operatorname{det}\left[\lambda I-A_{0}-A_{1} \exp (-\lambda r)\right] \\
& =\operatorname{det}\left[\begin{array}{cc}
\lambda & -1 \\
g_{y}(0) \exp (-\lambda r) & \lambda+f(0)
\end{array}\right] \\
& =\lambda^{2}+\lambda f(0)+g_{y}(0) \exp (-\lambda r) .
\end{aligned}
$$

Let $\omega \in \mathbb{R}$. Then $d(j \omega)$ vanishes iff

$\operatorname{Re} d(j \omega)=-\omega^{2}+g_{y}(0) \cos (-\omega r)=0$

i.e.,

$$
\omega^{2}=g_{y}(0) \cos (\omega r)
$$

and

$\operatorname{Im} d(j \omega)=f(0) \omega+g_{y}(0) \sin (-\omega r)=0$

i.e.,

$$
\omega=g_{y}(0) / f(0) \sin (\omega r) .
$$

Conditions (3.9) and (3.10) are satisfied only at finitely many points, excluding in some cases - for fixed values of $g_{y}(0), f(0)$, and $r$ certain frequencies w. Then assumption (a) of Theorem 1 is satisfied. Furthermore, assumptions (b) and (c) hold because of condition (3.6). In the following $\mathrm{H}_{\mathrm{x}}, \mathrm{H}_{\mathrm{xx}}, \mathrm{H}_{\mathrm{xy}}$, etc. denote first and second derivatives of $H(x, y, u, \lambda)$, evaluated at $\left(x^{0}, y^{0}, u^{0}, \lambda\right)$, with $x^{0}=0, y^{0}=0, u^{0}=0$ and $\lambda$ given by $(3.7)$.

$$
\begin{aligned}
& \text { We find (cp. [3]) } \\
& \begin{array}{l}
P(w)=H_{x x}+2 H_{x y} \exp (-j w r)+H_{y y} \\
Q(w)=H_{u x}+H_{u y} \exp (-j u r) \\
R=H_{u u} .
\end{array}
\end{aligned}
$$


One easily sees

$$
H_{x y}=0, \quad H_{u x}=0, \quad H_{u y}=0, \quad H_{u u}=\alpha .
$$

Hence (cp. (2.12))

$$
\pi(\omega)=B^{T} \Delta^{-1}(-j \omega)^{T} P(\omega) \Delta^{-1}(j \omega) B+\alpha
$$

and

$$
P(\omega)=P=H_{x x}+H_{y y} .
$$

We compute, taking into account (3.6)

$$
\begin{aligned}
& H_{x x}=\left(\begin{array}{cc}
0 & g_{y}(0)^{-1} f_{x}(0) \\
g_{y}(0)^{-1} f_{x}(0) & 0
\end{array}\right) \\
& H_{y y}=\left(\begin{array}{cc}
g_{y}(0)^{-1} g_{y y}(0) & 0 \\
0 & 0
\end{array}\right) .
\end{aligned}
$$

Thus

$$
P=g_{y}(0)^{-1}\left(\begin{array}{ll}
g_{y y}(0) & f_{x}(0) \\
f_{x}(0) & 0
\end{array}\right) \text {. }
$$

For the transfer function of (3.8) we obtain

$$
\begin{aligned}
& \Delta^{-1}(\lambda) B \\
& =d^{-1}(\lambda)\left[\begin{array}{ll}
\lambda+f(0) & 1 \\
-g_{y}(0) \exp (-\lambda r) & \lambda
\end{array}\right]\left[\begin{array}{l}
0 \\
1
\end{array}\right] \\
& =d^{-1}(\lambda)\left[\begin{array}{l}
1 \\
\lambda
\end{array}\right] .
\end{aligned}
$$

Since

$$
d(-j \omega) d(j \omega)=\left(\operatorname{Re} d(j \omega)^{2}+(\operatorname{Im} d(j \omega))^{2}\right.
$$

we find 


$$
\begin{aligned}
e(\omega):=d(-j \omega) d(j \omega)= & \left(\omega^{2}-g_{y}(0) \cos (\omega r)\right)^{2}+(\omega f(0) \\
& \left.-g_{y}(0) \sin (\omega r)\right)^{2} .
\end{aligned}
$$

All this in (3.11) yields

$$
\begin{aligned}
\Pi(\omega)= & g_{y}(0)^{-1} g_{y y}(0) /\left[\left(\omega^{2}-g_{y}(0) \cos (\omega r)\right)^{2}+(\omega f(0)\right. \\
& \left.\left.-g_{y}(0) \sin (\omega r)\right)^{2}\right]+\alpha .
\end{aligned}
$$

The $\pi$-Criterion (Theorem 1 above) asserts that $\left(x^{0}, u^{0}\right)$ is locally proper if this matrix is partially negative.

\section{Discussion of $\pi(\omega)$ and Numerical Results.}

Let us first consider the easy linear case where

$$
g(y)=\text { ay, } \quad a \neq 0 \text {. }
$$

This comprises in particular the retarded Van der Pol equation

$$
\ddot{x}(t)+\left(x(t)^{2}-x\right) \dot{x}(t)+x(t-r)=u(t) .
$$

The assumptions $(3.4)$ and $(3.6)$ are satisfied and $\varepsilon_{y y}(0)=0$. Hence

$$
\Pi(\omega)=\alpha,
$$

and we have no assertion for $\alpha \geq 0$, while local properness holds for $\alpha<0$, e.g. for the performance criterion

$$
\text { minimize }-1 / \tau \int_{0}^{\tau} x_{1}(s) d s-1 / \tau \int_{0}^{\tau} u(s)^{2} d s
$$

for all periods $\tau>0$.

Using this criterion, we maximize the average output plus the control energy. Now under the assumptions of Theorem 2, in particular in the case $a<1$, every periodic control $u$ with $\int_{0}^{T} u(s) d s=0$ generates a periodic trajectory. Clearly, every non-constant periodic control has positive energy $\int_{0}^{T} u(s)^{2} d s$. Hence the result above is clear. More interesting is 
the nonlinear case where $g_{y y}(0) \neq 0$. Let us first consider the unretarded equation i.e. $r=0$.

Then by (3.12)

$$
\begin{aligned}
e(\omega) & =\left(\omega^{2}-g_{y}(0)\right)^{2}+(\omega f(0))^{2} \\
& =\left[\omega^{2}-g_{y}(0)+1 / 2 f(0)^{2}\right]^{2}+g_{y}(0) f(0)^{2}-1 / 4 f(0)^{4} .
\end{aligned}
$$

Thus $e(\omega)=0$ iff

$$
\omega^{2}=g_{y}(0)-1 / 2 f(0)^{2} \pm f(0) \sqrt{g_{y}(0)-1 / 4 f(0)^{2}} .
$$

If e.g. $g_{y}(0)>0, f(0)=0$, then the only positive root of $e(\omega)$ is $\sqrt{\mathrm{g}_{\mathrm{y}}(0)}$ and one has for every a that

$$
\Pi(\omega)=g_{y}(0)^{-1} g_{y y}(0) e(\omega)^{-1}+\alpha
$$

will have a negative pole if $\mathrm{g}_{\mathrm{y}}(0) \mathrm{g}_{\mathrm{yy}}(0)<0$ (observe that always $e(\omega) \geq 0)$.

In the following, we let

$$
\mathrm{g}_{\mathrm{y}}(0)=1, \quad f(0)=0
$$

In this case, we can simplify the expression for $\pi(\omega)$ considerably. From (3.12)

$$
\begin{aligned}
e(\omega) & =\left(\omega^{2}-\cos (\omega r)\right)^{2}+\sin ^{2}(\omega r) \\
& =\omega^{4}-2 \omega^{2} \cos (\omega \mathbf{r})+1 .
\end{aligned}
$$

Hence by (3.13)

$$
\Pi(\omega)=\varepsilon_{\mathrm{yy}}(0) /\left[\omega^{4}-2 \omega^{2} \cos (\omega \mathrm{r})+1\right]+\alpha .
$$

Consider the case $\alpha=0$ and suppose $g_{y y}(0)<0$. Then always $\pi(\omega)<0$ and local properness holds. This can easily be explained as in the linear case above.

More complicated in the case $\alpha=1$, where one wants to minimize

$$
-1 / \tau \int_{0}^{\tau} x(s) d s+1 / \tau \int_{0}^{\tau} u(s)^{2} d s .
$$


Here two effects are working against each other: while the average "output" can be increased by allowing for periodic controls, a certain price for the necessary "control energy" has to be paid.

Figures 1 - 8 below show $\Pi(\omega)$ for the parameter values

$$
\mathrm{g}_{\mathrm{yy}}(0)=-1 \text { and } \alpha=1
$$

and different values of the retardation $r$, starting with $r=0$.

For $r=0$, there is a pole at $\omega_{0}=1$ and for low frequencies $\omega$ local properness occurs, i.e., performance can be improved by allowing for periodic solutions. A good first guess for the optimal frequency would be close to $\omega_{0}$. In fact, chemical engineers use the $\pi$-Criterion in this way in order to determine suboptimal periodic controls (see e.g. [5, p. 1158]). If the retardation $r$ is increased, the pole vanishes; however, the behaviour with respect to local properness remains qualitatively unchanged.

If the retardation $r$ is further increased there appear new zones of properness, and their number increases as the retardation increases. If e.g. $r=100$, it appears very hard to draw any reasonable information about the location of the optimal period length out of the I-Criterion. Furthermore, the problem apparently becomes very sensitive with respect to the period length (at least, for sinusoidal inputs).

The conjecture is that, with increasing retardation the number of local minima increases. These remarks emphasize the need to find efficient criteria for the determination of the optimal period length.

\section{Acknowledgement.}

I thank Norbert Matzl ror his help in producing the computer plots.

\section{References.}

[1] Barbanti, L.: Liênard equations and control, Functional Differential Equations and Bifurcation, A.F.Izé, ed., Lecture Notes in Math., Vol. 799, Springer-Verlag, 1980, 1-22.

[2] Colonius, F.: Optimality for periodic control of functional differential systems, Report No. 36-1984, Mathematisches Institut fer Universität Graz, Graz 1984, submitted to J. Math. Anal. Appl.

[3] Colonius, F.: The high-frequency n-Criterion for retarded systems, Report No. 37-1984, Mathernatisches Institut der Universität Graz, Graz 1984, submitted to IEEE Trans. Aut. Control. 
(4) DePascale, E. and Iannacci, R.: Periodic solutions of generalized Liénard equations with delay, Equadiff $82, \mathrm{H} . \mathrm{W}$. Knobloch and K. Schmitt, eds., Springer-Verlag 1983, $148-156$.

[5] Sincic, D. and Bailey, J.E.: Analytical Optimization and Sensitivity analysis of forced periodic chemical processes, Chem. Eng. Sci., 35 $(1980), 1153-1165$.

Appendix.

The following figures $1-8$ show the curves $\Pi(\omega)$ for different values of the retardation $r$. Here $\Pi(\omega)$ is determined by (4.1) and (4.3), i.e., the parameter values are

$$
f(0)=0, \quad g_{y}(0)=1, \quad g_{y y}(0)=-1, \quad \alpha=1
$$

and

$$
\pi(\omega)=1-1 /\left[\omega^{4}-2 \omega^{2} \cos (\omega r)+1\right] .
$$

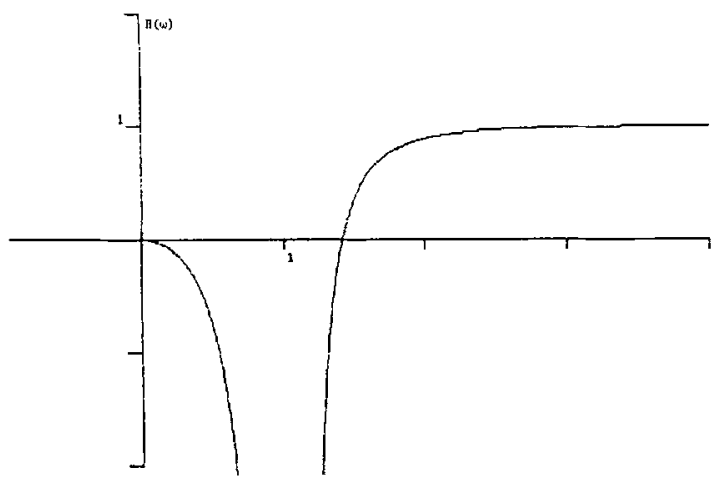

Figure 1: $r=0.00$

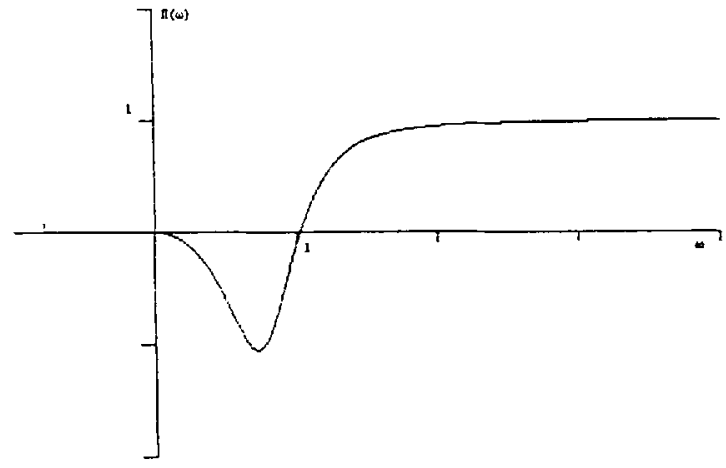

Figure 2: $r=1.00$ 


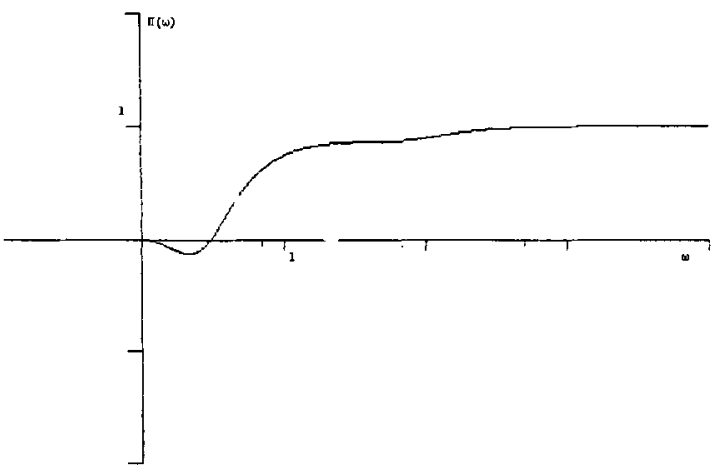

Figure 3: $r=3.00$

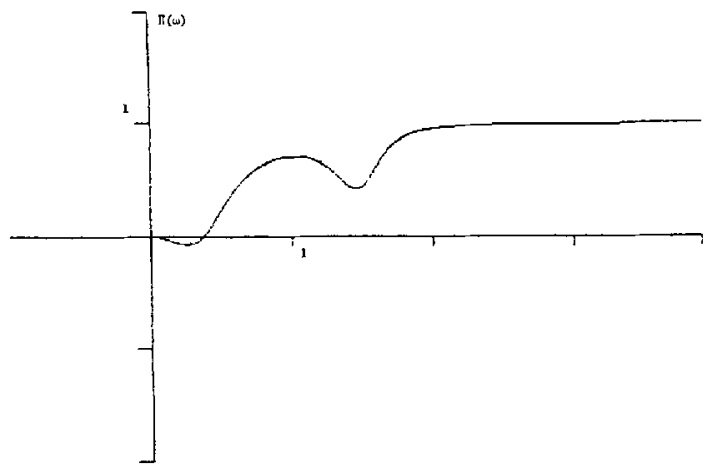

Figure 4: $r=4.00$

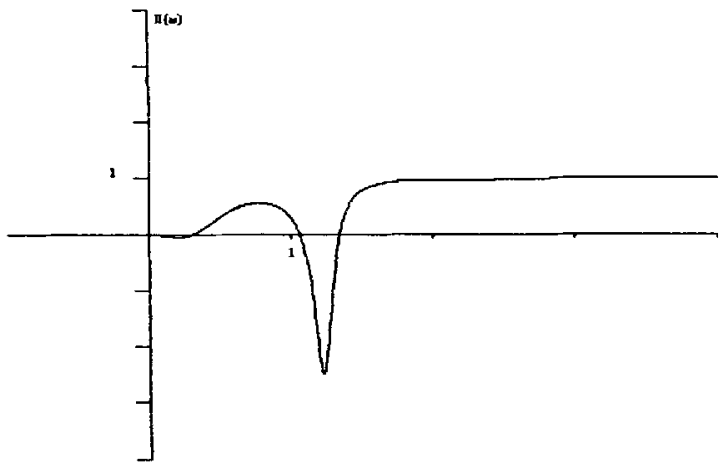

Figure 5: $r=5.00$ 


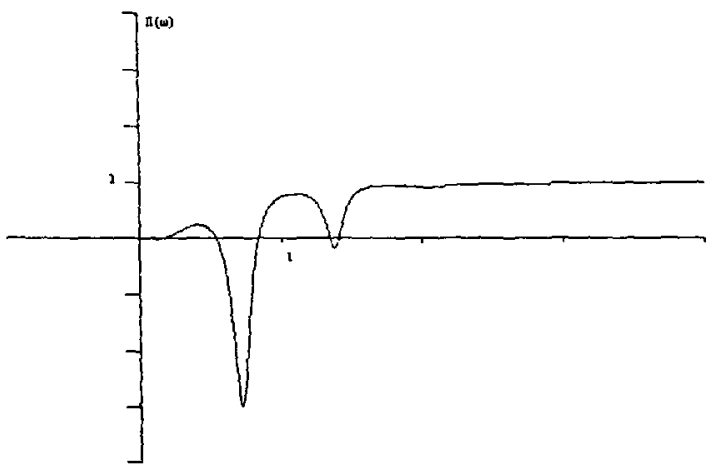

Figure 6: $r=9.00$

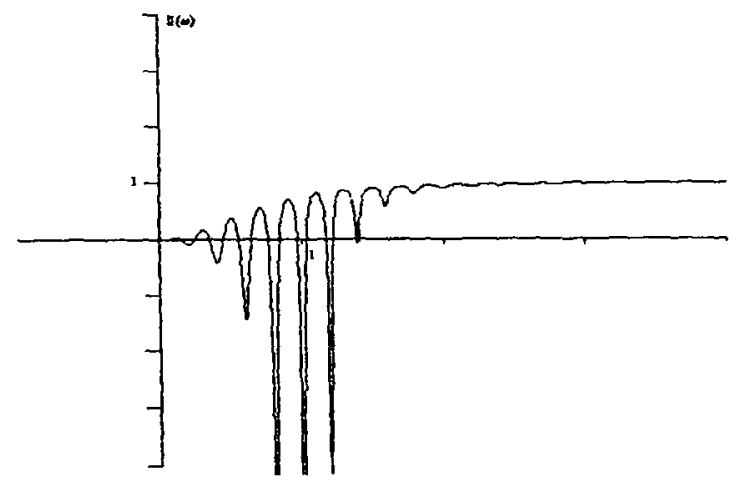

Figure $7: r=31.40$

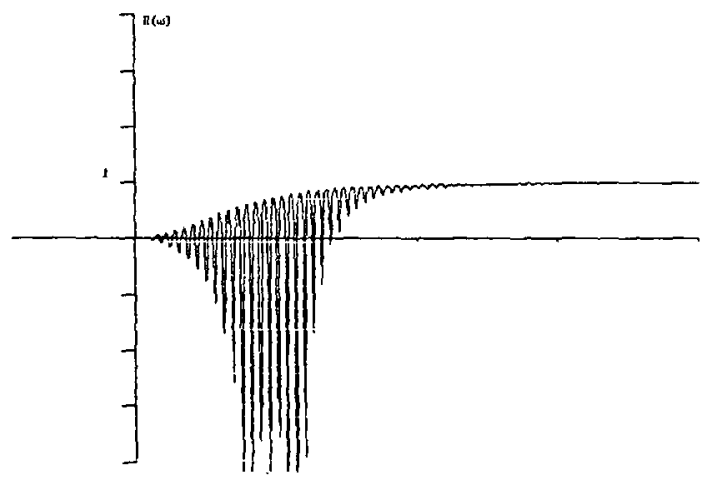

Figure $8: r=100.00$ 